\title{
La Fiction en école d'ingénieurs : écrire et lire autrement
}

\author{
Véronique QUANQUIN veronique.quanquin@polytech.univ-bpclermont.fr \\ Monique RODENBURG monique.rodenburg@polytech.univ-bpclermont.fr \\ Claire RENARD claire.renard@polytech.univ-bpclermont.fr \\ Polytech'Clermont Ferrand \\ Campus des Cézeaux \\ 24, Avenue des Landais \\ BP 206 \\ 63174 AUBIÈRE CEDEX FRANCE
}

RESUME : Dans le cadre de l'enseignement de l'expression et de la communication en école d'ingénieurs deux expériences pédagogiques ont été mises en place : lecture de romans en deuxième année de génie électrique et écriture de nouvelles pour l'ensemble des étudiants de tronc commun. Elles ont pour objectif de développer les compétences langagières des étudiants par le biais de la Fiction. Ces expériences ont montré que les étudiants peuvent développer leur créativité et s'impliquer activement dans une démarche culturelle.

\section{Mots Clés : fiction, écriture et lecture, créativité, public scientifique}

\section{INTRODUCTION}

Polytech'Clermont Ferrand est une école d'ingénieurs en 3 ans qui recrute aussi bien des étudiants de classe préparatoire que des étudiants venant de DUT, Deug, BTS et Formation Continue ainsi que des étudiants étrangers parfois nonfrancophones. Les étudiants des 5 départements Génie Civil, Génie Biologique, Génie Electrique, Génie Mathématique et Modélisation et Génie Physique auront tous les mêmes besoins en terme d'Expression, Communication et Cultures lorsqu'ils seront en entreprise, mais ne possèdent pas tous les mêmes compétences lorsqu'ils intègrent notre école.

Ce constat nous a amené à concevoir, à l'intérieur d'une philosophie générale détaillée en partie I, des approches adaptées à chaque département et modulées en fonction de besoins plus spécifiques.

Un des atouts de l'école est qu'elle partage avec une autre école du campus un CDI et deux documentalistes. Ce CDI ne possédait que des ouvrages et revues techniques et scientifiques jusqu'en 2002, l'autre bibliothèque du campus est elle aussi scientifique. Il a semblé intéressant d'élargir à la fiction le type d'ouvrages accessibles dans nos locaux et de renforcer la fréquentation du CDI grâce à une collaboration plus forte entre enseignants et documentalistes.

Est aussi apparu le besoin d'apporter aux étudiants une pratique de la langue qui ne soit pas uniquement technique ou scientifique, une pratique qui leur ouvre des horizons différents, qui les amène à savoir parler d'autre chose.

Après avoir présenté la philosophie qui soustend nos enseignements d'Expression, Communication et Cultures, nous présenterons les 2 projets pédagogiques basés sur la Fiction : le " Projet lecture » en place depuis 2002 et le «Concours de Nouvelles» en place depuis 2008 .

\section{EXPRESSION COMMUNICATION ET CULTURES : PRESENTATION}

Les cours d'Expression, Communication et Cultures (E2C) à Polytech'Clermont-Ferrand ont pour objectif d'amener les étudiants à développer une pensée autonome, à s'ouvrir au monde de l'entreprise et à appréhender des univers culturels les plus divers possibles. Pour ce faire, les cours s'articulent autour de trois axes principaux : l'Expression Ecrite et Orale, la Communication et les Cultures. Les méthodologies de travail pour ces 3 axes comprennent différents travaux à réaliser individuellement ou en groupe et dans lesquels la créativité tient un rôle important.

\subsection{Expression écrite et orale}

D'un point de vue général, il s'agit, pour les étudiants, d'auto-évaluer leurs compétences afin de développer et améliorer leur expression écrite et orale tout en ciblant leur public et en déterminant le meilleur support possible pour transmettre l'information.

Les exercices-supports sont : techniques d'exposé enseignées et mises en pratique au cours d'exposés courts ou longs, en temps limité ou non, classiques ou mis en scène par exemple. Sont aussi proposés : techniques d'écriture incluant la vulgarisation, rédaction d'articles, exercices d'argumentation, simulation de situations de communication etc.

L'expression écrite et orale est aussi travaillée dans le but de maîtriser les outils nécessaires aux études supérieures et au travail en entreprise : écriture 
de rapport de stage ou de projet, soutenance, compte rendu oral ou écrit d'un travail à un public donné (enseignants des disciplines, hors disciplines ou entreprises par exemple), recherche d'information, validation des sources et synthèse de documents.

Enfin, les techniques d'expression écrite et orale sont mises au service de la recherche d'emploi : rédaction de $\mathrm{CV}$, lettres, préparation à l'entretien, valorisation de l'expérience...

\subsection{Communication}

L'objectif est de prendre conscience qu'il existe des méthodes et outils permettant d'améliorer sa propre communication, que communiquer correctement ne relève pas du hasard mais s'appuie sur l'acquisition de connaissances théoriques, la mise en pratique de ces connaissances et l'analyse de situations de communication.

Le bagage théorique comprend entre autres les points suivants : modèle mathématique de la communication, Palo Alto, langage et communication, verbal-non verbal-paraverbal, analyse transactionnelle...

Les étudiants feront aussi des simulations (activités théâtralisées), des analyses de situations de communication vécues (stage, vie professionnelle, études etc.) ou non (extraites de livres ou films par exemple). Enfin, la communication d'entreprise sera étudiée à partir des journaux d'entreprise, expériences, ou rencontres avec des professionnels.

\subsection{Cultures}

L'ingénieur ne peut plus être seulement un super-technicien. Il a besoin de s'ouvrir et d'avoir une vision globale des gens, de la société, de l'entreprise et du monde. Faire état d'une curiosité concernant le monde de l'entreprise, montrer qu'on est intéressé par l'actualité et qu'on lit la presse, savoir parler d'une exposition de peinture, d'un concert, du livre que l'on a lu, n'est-ce pas cela qui fait la différence aussi bien à l'embauche qu'en entreprise?

Le travail sur cet axe inclue une réflexion sur la place des cultures dans la communication par le biais de revues de presse culturelles ou économiques, conception d'affiches, rencontres et dialogues avec le monde de l'entreprise : ces exercices et d'autres encore sont proposés pour construire un savoir/savoir-faire complémentaire au savoir scientifique.

\section{PROJET LECTURE EN ELECTRIQUE DEUXIEME ANNEE}

GENIE 2.1 Problématique
Les étudiants de ce département viennent majoritairement de filières techniques et leur maîtrise de l'expression orale et écrite est parfois aléatoire. Or les besoins dans ces domaines sont grands de la recherche d'emploi à la gestion de clients, d'une équipe ou d'un service en entreprise.

Dans le cadre des enseignements techniques, ces étudiants mènent un projet de type industriel en binôme en deuxième et troisième année qui donne lieu à une soutenance, à l'écriture d'un rapport et à la réalisation d'un poster et ils ont donc besoin d'améliorer leurs capacités à rendre compte d'un travail.

C'est pourquoi en collaboration avec les documentalistes, le projet lecture a été créé en 2002 . Il s'agit de développer des savoir-faire mais avec un langage non technique, il s'agit aussi de faire découvrir ou redécouvrir le plaisir de lire.

\subsection{Public}

Etudiants de Génie Electrique en deuxième année (avant dernière année). Volume horaire par étudiant $3 \mathrm{~h}$ sur $20 \mathrm{~h}$ plus travail personnel. Travail compris dans le curriculum.

\subsection{Description du projet}

Les étudiants en binôme, après présentation du thème qui change tous les ans, choisissent un roman dans la liste. Le thème pour 2009-2010 est « Qui suisje?»

Les romans contemporains, les auteurs de toutes origines et nationalités sont privilégiés.

Pendant l'été, les romans sont lus par les documentalistes, l'enseignante en communication de GE2 et des collègues scientifiques amateurs de lecture ce qui permet une collaboration transdisciplinaire et un échange réel avec les étudiants lors du choix du roman.

Les romans sont achetés par l'école en 2 exemplaires et prêtés de Septembre, lors du premier cours, à Février lors de la présentation orale. A la fin du travail, ils viennent alimenter le fond du CDI.

Les étudiants ont un mois pour commencer à lire, changer d'avis et faire un choix final ce qui les amènent à aller au CDI et à discuter avec les documentalistes. Ils sont placés dans une vraie situation de lecteur avec la possibilité de faire des choix.

Le travail est présenté lors du Cours 1/9. Les soutenances ont lieu lors des Cours 8/9 et 9/9 et le jury est composé de l'enseignante, d'une des documentalistes, d'enseignants de matières scientifiques, ce qui permet de démontrer que la lecture est transversale et non réservée aux littéraires.

\subsection{Description du travail}


Objectifs : savoir travailler en projet et en équipe, savoir rechercher des documents et de l'information, savoir citer des sources, savoir faire une bibliographie, savoir rédiger un rapport, s'entrainer à la présentation orale, savoir argumenter et débattre vis-àvis d'un auditoire.

Description : Elaboration d'un dossier/rapport, d'un poster de présentation de ce roman, et présentation orale en classe de l'ensemble. Les 3 composantes du travail sont notées ( 1 note d'oral +1 note d'écrit +1 note poster)

\subsubsection{Présentation orale : 1 par groupe}

La présentation orale dure de 15 à 20 minutes par binôme et comprend :

La présentation du contenu du rapport ;

L'expression de son opinion personnelle sur l'œuvre en argumentant ;

La lecture d'un très court passage qui semble particulièrement marquant ;

La justification des choix en ce qui concerne le poster.

Elle est suivie d'une session de 5 à 10 minutes de réponse aux questions du groupe et du professeur.

\subsubsection{Poster : 1 par groupe (Format A3)}

L'esprit de ce travail est de présenter l'essence, l'essentiel du livre. On peut se référer à ce qu'est un poster de film. Le poster comprend du visuel, de la couleur, le titre et le nom de l'auteur. Il comprend aussi soit des mots clés, soit une phrase interrogative qui peut reprendre la problématique du livre, soit un court texte. La cible (l'interlocuteur ou les interlocuteurs) est les étudiants et les personnels du CUST. Le poster doit donner une image plutôt juste de ce qu'est le livre lu et doit donner envie de lire ce livre. Les posters sont ensuite affichés au CDI du CUST où les livres pourront être empruntés.

\subsubsection{Dossier : 1 par groupe (à rendre}

\section{au professeur)} suivantes :

Le dossier doit comporter au moins les parties

-biographie courte de l'auteur

- résumé du livre

- personnages principaux

- thème(s) abordé(s) et/ou problématique soulevée par l'auteur et comparaison avec la/les version(s) filmique(s) dans la mesure du possible

- collection d'articles de presse, de critiques sur le roman (en anglais si aucun article français n'est disponible). Cette collection d'articles est placée en annexe et fait l'objet d'une synthèse au début de l'article personnel.

-article personnel et critique sur le livre : réflexion sur le titre et son sens (est-il différent du titre dans la langue d'origine ?), synthèse des articles, ce que le roman apporte et/ou apprend, avis personnel ...

Pour clore le travail et le valoriser, un vernissage de l'exposition des posters a lieu au CDI en Mars en présence des autres étudiants et des personnels de l'école.

Un article personnel de 2 pages manuscrites est à rédiger après lecture d'un deuxième roman en début de GE3.

\subsection{Bilan}

Une fois le premier choc passé et les mauvais souvenirs de lycée oubliés pour certains, les étudiants se prennent au jeu et valident le travail fait lors de ce projet qui pour le rendu correspond exactement au projet industriel.

Lors des séances de soutenances, les étudiants font preuve d'une qualité d'écoute assez exceptionnelle et d'un intérêt réel pour ce qui est présenté. Les présentations même si elles sont de qualités inégales sont toujours faites très sérieusement. Les documentalistes, des enseignants de Génie Electrique, d'autres enseignants de communication participent aux soutenances, ce qui montre l'intérêt porté à ce travail et le valorise. Chaque année des étudiants disent que ce projet a été un déclencheur de lecture, qu'ils n'ont pas pu poser le livre, que c'est « leur premier roman »! En ce qui concerne la réalisation des posters, ils développent des compétences au niveau créatif et au niveau de l'abstraction et apprennent à communiquer l'essentiel en tenant compte d'un public donné, ce qui est précieux pour un ingénieur. Lors des évaluations des enseignements, la validité de ce travail est confirmée par les étudiants y compris par les étudiants étrangers francophones.

$\mathrm{Au}$ niveau du CDI, des échanges autour des romans de type lecteur/lecteur entre les étudiants et les documentalistes se sont créés. Les étudiants viennent plus volontiers faire des recherches sur place. Ils s'intéressent aux romans présentés par d'autres, les empruntent, demandent d'autres titres. Ils développent une curiosité intellectuelle et ne s'interdisent plus de lire. En apprenant à s'exprimer sur d'autres sujets que la technique ou la science ils acquièrent une aisance et une ouverture d'esprit qui leurs seront utiles dans leur futur métier d'ingénieur.

\section{CONCOURS DE NOUVELLES}


Faire écrire des étudiants est toujours une tâche difficile à mettre en œuvre en raison principalement de ce qu'écrire ne peut être qu'un acte individuel. Pendant des TP ou des TD, une des seules pratiques possibles est celle de l'atelier d'écriture (Haddad, 2006) ; il est alors indispensable de fixer un but à cet atelier. Participer à un concours de nouvelles est un but qui peut être stimulant. Cette action a été initiée pendant l'année scolaire 2008-2009, elle est reconduite pour cette nouvelle année qui commence.

\subsection{Etudiants}

Le concours a été proposé à tous les étudiants de première année des écoles Polytech'Clermont Ferrand et ISIMA. Il faut signaler que ce concours est un peu particulier car il est obligatoire et à double impact. Chaque étudiant doit écrire une nouvelle qui fera d'une part l'objet d'une notation, intégrée dans les notes de Communication. D'autre part, les nouvelles sont toutes proposées au concours, des jurys décident alors des prix qui leur seront ou non attribués.

\subsection{Besoins}

Il est rapidement demandé aux étudiants, dans leur cursus, de rédiger des rapports de projet ou de stage ou de faire des comptes rendus d'expérience. Commencer par une écriture fictionnelle permet de s'intéresser à des savoirs et savoir-faire langagiers de base comme la cohérence d'un texte ou l'utilisation des temps verbaux. Pour certains, il s'agira de révision, pour d'autres d'une remise à niveau. Nous partons de l'hypothèse que ce qui est travaillé dans ce contexte est transférable aux écrits scientifiques étant donné les deux compétences nécessaires pour rédiger ces écrits : une compétence concernant le domaine, et une compétence langagière, d'expression écrite. Diversifier les types d'écrit permet de diversifier les approches pédagogiques en montrant l'importance d'une expression écrite correcte.

Le travail sur la langue écrite est de plus indispensable pour certains étudiants présentant des difficultés par rapport à cette compétence, en particulier les étudiants non francophones.

Enfin, ce travail d'écriture fictionnelle, parce qu'il déstabilise des étudiants plutôt habitués à rédiger des écrits scientifiques, les conduit à être créatifs, à travailler leur créativité, ce qui ne peut être qu'un atout dans l'emploi et la recherche d'emploi.

\subsection{Modalités de réalisation}

Le même thème a été donné, en début d'année, à tous les étudiants ; il s'agissait pour cette année du thème «Terre(s) ». Un document leur a été distribué comprenant outre des nouvelles d'auteurs classiques, des conseils de rédaction émanant des enseignants. Certains collègues écrivent eux-mêmes, ou sont de grands lecteurs ou encore ont été enseignants en lycée, par conséquent les conseils qui ont été donnés varient et traitent de la nouvelle en tant qu'écrit codé ou bien de l'acte d'écrire lui-même.

Pour qu'il y ait égalité de tous les étudiants devant ce travail, il a été décidé de faire 2 ou 3 TD de travail en groupe de 2 heures chacun, rien de plus. Chaque enseignant a alors travaillé comme il le souhaitait : lecture et analyse de nouvelles (Rey, 2001), travail sur les personnages, création de scénario, écriture en groupe, relecture, analyse collective des nouvelles écrites, etc. A chacun d'inventer la façon de faire travailler les étudiants. Une seule contrainte était imposée : ne pas travailler avec les étudiants sur leur propre nouvelle.

Les nouvelles ont été livrées anonymement, sous forme de fichier informatique ; chaque étudiant a alors signé une charte indiquant le numéro de code de la nouvelle. Grâce à ce procédé, aucun des membres de jury n'a pu avoir accès aux noms des auteurs, la lecture et la notation sont donc totalement anonymes. Les jurys, composés de deux personnes issues des deux écoles, ont attribué les notes et proposé des nouvelles au concours. Ainsi, une première sélection, relativement importante a-t-elle été faite puisque des 300 nouvelles initiales, environ 60 ont été proposées pour le deuxième jury.

La charge du deuxième jury étant d'attribuer des prix, il a été limité à 6 personnes, la bibliothécaire, des enseignants d'Expression et Communication et des personnels administratifs, chacune ayant été membre des jurys précédents. La démarche a été la suivante : après une lecture individuelle des nouvelles présélectionnées, le jury a procédé à un vote pour chacune d'elles. Ce vote est à la majorité, donc à chaque membre de défendre ses choix, d'argumenter, de convaincre ses collègues ; ce vote à la majorité suppose aussi d'accepter que ses propres choix ne «passent » pas, donc de faire des concessions. Si le premier jury a nécessairement un fonctionnement plutôt scolaire, le deuxième est plus calqué sur les jurys de grands prix, Goncourt ou Renaudot, pour ne citer qu'eux.

Les prix décernés sont au nombre de 6. D'un point de vue local, il a été question du prix du scénario, de l'imaginaire, du dénouement et de l'écriture ; une " palme d'or » a été décernée ; enfin la bibliothécaire, parce qu'elle représente, par sa fonction, la lecture donc plus largement la culture, a décerné un prix spécifique. Ces différentes récompenses mettent bien en valeur tout ce qui fait d'une production écrite, un écrit satisfaisant : un plan correct, une créativité pertinente, une clôture du texte et enfin une écriture au moins correcte sinon travaillée.

Les prix ont été remis lors d'une cérémonie officialisant le concours, et en présence des étudiants, 
des jurys, des directions des écoles et de toutes les personnes intéressées par cette action.

\subsection{Ce qui n'avait pas été prévu}

Comme dans toute nouvelle action pédagogique, tout semble avoir été prévu, la réalité est tout autre. Deux problèmes principaux se sont posés, le plagiat ou vol intellectuel et la liberté thématique.

Certaines nouvelles ont rappelé aux lecteursjurys d'autres écrits. Un système de discussion et d'échange a donc été mis en place, afin de mutualiser les connaissances littéraires, et beaucoup de personnes extérieures aux jurys ont été sollicitées. Car il ne s'agit pas simplement de trouver une ressemblance entre des écrits, encore faut-il pouvoir prouver qu'il s'agit d'un plagiat en trouvant le texte originel. Cette attitude de pillage, largement facilitée par la mise à disposition de beaucoup de documents au moyen d'internet, pose le problème de l'honnêteté intellectuelle qui dépasse largement le cadre d'un travail scolaire. Ces constatations ont conduit à modifier la charte en incluant une clause qui indique que si le plagiat est avéré, la note zéro serait attribuée à la nouvelle qui par ailleurs ne pourrait concourir à un prix. Enfin, un écrit « en classe » sera réalisé par tous les étudiants; si la comparaison entre cet écrit et la nouvelle pose des problèmes, le jury final se réserve le droit de ne pas proposer aux prix un texte qui ne semble pas avoir été produit par l'auteur. Ainsi, le problème de la preuve du plagiat se trouve-t-il contourné.

L'autre problème posé par les écrits des étudiants et qui a été sujet de nombre de discussions est celui de la liberté d'écriture. Peut-on laisser toute liberté d'écriture aux étudiants, dans un exercice pédagogique, intégré à un travail d'école ? Doit-on limiter la production de textes érotiques ou violents par exemple ? Dans une situation d'écriture fictionnelle quelconque, il ne peut y avoir censure, l'auteur est libre ; il peut ne pas être édité, mais rien ne l'empêche d'écrire ce qu'il souhaite. Concernant ce concours, la décision a été prise de ne pas censurer ; cependant, une association culturelle inter-école va être mise en place, elle permettra de sortir le concours du cadre de formation et par conséquent de ne pas avoir d'exigences scolaires trop fortes.

\subsection{Bilan et perspectives}

Retour des étudiants sur le concours de nouvelles.

Nous n'avons pas fait une enquête systématique, mais recueilli des avis d'étudiants primés; de plus des discussions/bilans ont été menées en cours avec tous les participants. Il en ressort que les élèves ingénieurs, par conséquent scientifiques, ont apprécié cet exercice conduisant à la fois à faire un travail différent en TD (analyse de nouvelles et atelier d'écriture) et une réalisation peu habituelle. Les étudiants mettent en évidence le fait que cet exercice demande beaucoup de travail personnel; ils évoquent aussi le plagiat, en particulier non avéré, en soulignant l'injustice dans l'évaluation, plus particulièrement quand ils ont euxmêmes réalisé « honnêtement » cet exercice. Enfin, la gratification par des prix (un livre remis, une attestation du prix et une invitation au restaurant pour tous les vainqueurs et les organisateurs de la manifestation) est très importante, cela marque le fait que l'on sort d'un exercice classique d'Expression et Communication pour vivre une véritable expérience de concours littéraire. Ornella (primée pour « En passant») conclut son évaluation de l'exercice en disant : «J'ai beaucoup aimé ce concours et je vous remercie encore de l'avoir organisé. J'espère vraiment que vous continuerez longtemps encore !».

Les étudiants ont « joué le jeu » dans l'immense majorité d'entre eux, l'ambiance à la remise des prix en étant un bon indicateur. Ne faire qu'un seul exercice d'écriture représente peu par rapport à ce qui pourrait/devrait être fait, mais il a permis, en plus des objectifs indiqués précédemment, de leur (re)donner confiance en leurs capacités d'expression, quel que soit le contexte.

Concernant les enseignants et personnels des écoles, la pluridisciplinarité mise en œuvre dans les jurys a initié de nombreuses et passionnées discussion, entre scientifiques et littéraires, enseignants et administratifs. Le partenariat inter-écoles a permis de construire de vrais échanges par rapport à un contenu enseignement/apprentissage, de plus il montre aux étudiants la cohérence des formations.

\section{CONCLUSION}

Les résultats positifs tant au niveau des étudiants que des écoles nous amènent à réitérer ces deux expériences qui montrent aux personnels et aux étudiants, la place de la communication dans des enseignements scientifiques.

\section{BIBLIOGRAPHIE}

GUIBERT Rozenn, Former des écrivants, Villeneuve d'Ascq, Presses Universitaires du Septentrion, 2003 HADDAD Hubert, Le nouveau magasin d'écriture, Zulma, 2006

REY Pierre-Louis, Le roman et la nouvelle, Profil Littéraire, Hatier, Paris, 2001 


\section{ANNEXES}

\section{Site d'accès aux nouvelles}

http://cust.univ-

bpclermont.fr/presentation/documentation/nouvelles/pa $\underline{\text { lmares.html }}$

\section{Concours de nouvelles}

\subsection{En PasSant, Ornella PERBET}

Le métro arrive, la foule se rapproche instinctivement de la plate-forme. Les portes blanches coulissent, quelques personnes descendent, elles sont immédiatement remplacées par celles qui attendaient sur le quai. Il est entrainé par leur flot, il avance, se fait repousser, résiste. Sa main attrape une poignée, il s'y arrime, les portes se ferment sans bruit. Autour de lui, de longues manches grises ondulent sous les pulsations du métro.

Premier arrêt.

La chaleur fait goutter les faces ternes, aucune ne le regarde; il étouffe.

Deuxième arrêt.

Encore quatre stations et il descendra, parc Medaga, rue Eguestin, deuxième à droite, 250 mètres, 64 marches. Une lasagne attend dans le frigo.

Troisième arrêt.

Par le parc, c'est plus rapide. Il met 4 min 30 de plus lorsqu'il passe par la rue Franche puis par le boulevard Adley. Une manche tombe sous une violente secousse. Un corps se balance puis en écrase d'autres. Bien sûr pour gagner ces 270 secondes, il doit marcher vite.

Quatrième arrêt.
Ses pieds se sont immobilisés, sa main raccrochée à une poignée salvatrice. Il n'a même pas sentit que c'était son bras qui tombait, son corps qui froissait les costumes. C'est Françoise qui lui avait montré la rue Eguestin, lui ne l'aurait jamais remarquée.

Cinquième arrêt.

Ses gestes sont automatiques. Françoise aime bien les petites ruelles, elle dit toujours qu'elles sont pleines de surprises, un peu comme elle. Lui préfère les boulevards et les avenues, il ne supporte pas l'odeur de la pisse.

Sixième arrêt.

Il descend, monte, il est dans le parc. Dans une semaine, il ne pourra plus le traverser.

Il a toujours aimé les arbres, leur façon d'être là, de pousser tous seuls, droits vers le ciel. Des enfants courent avec des nuages roses dans les mains, le vendeur de barbe-à-papa est encore ouvert. La belle maison de banlieue est pratiquement aménagée. Ils vont quitter l'appartement, à trois ils se montent dessus. Bientôt ils seront quatre, alors ils partent.

Il s'arrête et commande une géante. Les fils s'emmêlent sur le bâton. Il repart, le sourire aux lèvres, sa barbe-à-papa géante dans une main, son portedocument dans l'autre. A l'intérieur, les derniers contrats qu'il a réussit à négocier, son travail lui plaît ; il vient d'avoir une promotion.

Il s'installe sur un banc, regarde les enfants jouer sur le toboggan et dans le bac à sable en avalant sa barbe-à-papa. Dans le nouveau lotissement, il y a une aire de jeux pour les enfants. Dans une semaine, ce sera sa maison, sa pelouse, son garage, son break, ses voisins, son aire de jeux. Est-ce qu'il y a des arbres au moins ? Ils doivent être petits, tout juste des bâtons plantés dans le sol avec des fines feuilles au bout. Il 
préfère les grands arbres, vieux et sages. Les enfants rient, crient, trois d'entre eux chantent une comptine qu'il a oublié. Il fait beau, il a quitté sa veste et retroussé son pantalon.

Il mange cinq fruits et légumes par jour, fait plus de $30 \mathrm{mn}$ de sport quotidien, boit 1,5 1 d'eau minérale, ne fume pas, ne boit pas, ne conduit pas au-dessus de 90 $\mathrm{km} / \mathrm{h}$ sur route nationale et de 110 sur autoroute par temps de pluie. Employé dévoué, mari aimant et père de famille attentionné; Jean est un homme responsable.

Les parents le regardent hébétés. Tous enlèvent leurs enfants du bac à sable, les arrachent du toboggan et de la balançoire, arrêtent brusquement le tourniquet, tirent sur les bras et les mains. En quelques secondes, les pleurs et les cris couvrent la fuite et les regards furieux.

Il venait de finir sa barbe-à-papa et il mâchonnait tranquillement le bâton. Sans prévenir, soudain, il avait couru dans le bac à sable.

Maintenant, il est seul au milieu des dunes. Il rit en creusant le sable, des dizaines de trésors enfouis l'attendent. Le sable fin rentre dans ses chaussures, ses chaussettes, lui chatouille les orteils. Il creuse la terre en riant, s'excuse auprès de ses vieux amis de ne pas être venu les voir plus tôt. Il se dépêche ensuite de remplir ses poches : pierres rouges, galets difformes et vers de terre.

Il aperçoit le tourniquet. Une fois dessus, il le fait tourner à une vitesse supersonique : le bleu, le vert, le rouge et le jaune se mélangent jusqu'à donner des couleurs nouvelles aux teintes vives. Il veut courir vers elles. Sa tête tourne. Sa vision est floue. Il est aspiré à l'arrière, il tombe. A travers les barreaux de la cage à écureuil, de drôles de nuages défilent à toute vitesse. Un éléphant est avalé tout cru par une truite, un escargot arrive alors en courant pour lui venir en aide mais ils sont tous renversés par la moto gonflable. Il s'endort paisiblement, les arbres, le ciel et la Terre veilleront bien sur lui.

Quand il se relève, il fait presque nuit. Il a froid.

Il chasse minutieusement la poussière de sa chemise, récupère sa veste et son porte-document sur le banc. La lasagne l'attendra dans le frigo, ses mains sont sales, encore pleines de terre. Il quitte le parc. Il ne s'est pas retourné une seule fois.

\subsection{Plaie D'argent N'est Pas mortelle... Laura KREZALEK}

Benjamin Biolane est un homme cultivé, bien élevé. On peut dire qu'il a réussi sa vie. Il est cadre supérieur dans une grande société. Il possède une belle voiture, une jolie villa à l'extérieur de la ville, à la lisière de la forêt ou il aime faire son jogging. Il est assez beau garçon et pourtant il vit seul. Il a rencontré de nombreuses jolies filles dans son existence, mais tout comme ses amis, elles l'ont quitté les unes après les autres, car Benjamin a un énorme défaut : il est avare. Comment vous expliquer cela ? Il aime être invité à dîner, au cinéma...Mais il ne rend jamais l'invitation, ni aucun service gracieusement. Ramener un collègue chez lui coûte de l'essence, du temps, bref de l'argent, donc il préfère refuser poliment. Une collecte circule au bureau : il n'a jamais de liquide sur lui. Il ne boit aucun café à l'extérieur, le distributeur de l'entreprise est gratuit. Tous ces détails mis bout à bout, ont fait fuir le dernier de ses amis. En est-il conscient ? Il n'en semble pas affecté le moins du monde. Bien au contraire, il a décidé de faire installer une piscine dans son jardin. Il imagine déjà la soirée qu'il « donnera » quand les travaux seront terminés. Il demandera à chaque convive d'amener un plat, une boisson et lui se chargera de l'ambiance avec des lampions allumés tout autour de la piscine. Il en récupèrera pendant la retraite aux flambeaux organisée par la ville, ils sont gratuits. 
L'entreprise spécialisée en piscine lui a proposé deux devis. Un premier ou elle s'occupe de tout : terrassement, installation ; électricité, aménagement de finition et un deuxième avec installation minimaliste lui laissant le choix de l'entreprise de terrassement. Sans hésitation, il prit le deuxième devis. Il était tout excité et fier d'avoir trouvé la solution la plus économique pour creuser ce trou. Après tout, rien de bien compliqué ; il le ferait lui-même ! En louant une mini pelleteuse, il gagnerait facilement soixante pour cent du prix proposé par l'entreprise. Le voilà donc négociant la livraison gratuite de la pelleteuse afin de commencer les travaux dès le week-end prochain.

Le samedi à six heures du matin, il est à pied d'oeuvre, relisant une dernière fois la notice d'utilisation de l'engin. Il porte son plus vieux jogging, c'est parti, le premier coup de pelle est donné. Il jubile en pensant à la facilité de maniement des commandes Il va pouvoir travailler toute la journée, ainsi qu'une bonne partie de la soirée sans risquer de déranger les voisins. Les premiers sont à plus d'un kilomètre. Vers midi, il décide de se passer de déjeuner, plus vite il terminera, moins la location lui coûtera! Ce n'est que vers 17 heures qu'il s'autorise une pause pour se désaltérer et se dégourdir les jambes. Il a bien fait de prendre deux jours de congés, à ce rythme, il aura terminé suivant ses prévisions. Il fait le tour de ce trou béant. Il est assez satisfait de sa performance. Ces amas de terre tout autour ne l'affolent pas Il placera des dalles gravillonnées et après avoir étalé cette terre il sèmera $\mathrm{du}$ gazon. Les pluies de septembre le feront pousser rapidement. Si tout se déroule suivant ses plans, sa fête se fera le premier beau week-end d'octobre.

Il était dans ses pensées, quand son pied droit glissa. Il perdit l'équilibre, la terre meuble et glissante ne lui offrit aucune chance. Il essaya de s'accrocher avec ses mains en tombant au fond du trou.

C'est alors que l'imprévisible se produisit, un énorme pan de terre se détacha de la paroi, tout s'écroula. Remonter à tout prix !! Plus il se débattait, plus la terre le recouvrait. Cette odeur de terre humide qu'il aimait tant en foret, lui portait au coeur et le paniquait. Il avait de plus en plus de mal à respirer. Impossible de crier et surtout inutile. L'angoisse le gagnait, l'oxygène lui manquait.

Personne ne s'inquièterait de son absence avant mercredi. Il ne pouvait compter que sur lui-même.

Il essaya de fouiller ses poches pour attraper son portable. Quel réflexe inutile, de toute façon, il ne pouvait plus bouger ses bras. Il s'épuisait, après une telle journée ses forces le quittaient. Il avait envie de se reposer juste quelques minutes. Son cerveau sombrait dans un coma inévitable. Une de ses dernières pensées fut : dommage que je ne puisse rester ici, chez moi, avec une simple stèle au dessus de ma tête. Il va falloir payer une concession dans un cimetière !

\subsection{MONSIEUR PEPI Chloé GARREC}

Je m'appelle Margo, j'ai presque cinq ans et je suis en grande section maternelle. Mon école, c'est la plus grande de Pontivy. C'est aussi celle où les instituteurs sont les plus gentils. Moi, dans ma classe, j'ai deux maîtresses, et chacune d'entre elles est aidée par une assistante maternelle. Vous pensez sans doute que c'est beaucoup, mais il faut dire que nous sommes vraiment nombreux dans ma classe! Et c'est normal puisque mon école c'est la meilleure. C'est pour ça que Papa et Maman ont décidé que j'irai dans celle-ci. Mes parents font toujours ce qui est le mieux pour moi. Je les aime fort. Je n'ai jamais besoin de les attendre à la sortie de l'école, ils sont toujours à l'heure. Je les reconnais de loin avec leurs belles voitures rouges! Moi, à leur place, je n'aurais pas choisi la même couleur parce que d'accord c'est vrai qu'elles sont jolies leurs voitures rouges, mais moi j'aurais aussi aimé en avoir une comme celles de nos voisins. Elles sont bleues, ma couleur préférée, et tout le monde se retourne sur leur passage! Mais Papa n'a pas voulu. Il m'a expliqué qu'une seule voiture leur suffisait. Je suis d'accord, mais il aurait quand même pu choisir un autre modèle que celui de Maman. Ou Maman un autre 
modèle que celui de Papa! Bref, je ne sais pas qui a choisi en premier. Ils sont allés les acheter ensemble la semaine dernière, et quand je les ai vues garées toutes les deux devant la maison, si près l'une de l'autre, j'ai bien rigolé! Ils doivent s'aimer vraiment fort pour vouloir se ressembler autant.

Quand ma journée d'école est finie, je rentre à la maison avec Papa ou Maman. Ma maison aussi est grande et belle. J'y habite depuis que je suis née. Papa et Maman s'y sont installés peu de temps avant que j'arrive, pour avoir plus de place et surtout un grand jardin pour pouvoir installer la balançoire rouge. D'ailleurs en fait j'ai deux maisons. Elles ont été construites par la même personne, j'en suis sûre parce qu'elles sont identiques. La seule chose bizarre, c'est qu'elles sont côte à côte. Mais on n'habite que dans celle de droite. Celle de gauche, je n'y suis jamais allée. Papa et Maman la louent peut être. En tout cas, tout le monde doit avoir envie d'y habiter parce que ma maison, elle est géniale. Surtout ma chambre, elle est remplie de jouets! Je pourrais y rester toute la journée, et même encore plus depuis que le Père Noël m'a apporté les plus belles poupées du monde. Cette année j'ai été vraiment gâtée! C'est aussi la première fois où j'ai écrit ma lettre au Père Noël. Enfin, Maman m'a beaucoup aidé en écrivant les mots pour moi. Mais, avant de jouer, je dois faire mes exercices. C'est moins drôle mais Papa et Maman m'ont dit plusieurs fois que c'était très important pour moi que je les fasse. Mon préféré c'est le «Où est Charlie? » parce que c'est rigolo, mais il y en a plusieurs autres qui sont vraiment moins marrants et plus difficiles parce qu'il faut que je sois bien concentrée, et après une journée d'école c'est fatiguant! Ça va parce que ça ne dure jamais très longtemps et aussi parce que je ne les fais pas souvent toute seule. J'ai deux nourrices qui m'aident. Elles sont noires et se ressemblent énormément, pourtant maman m'a affirmé qu'elles n'étaient pas sœurs. Je les aime beaucoup, elles sont toujours très gentilles avec moi.

Aujourd'hui, je ne les verrai pas beaucoup parce que je dois aller avec Papa et Maman chez Monsieur Pépi. On va souvent lui rendre visite, environ deux fois par semaine. Par contre, je ne sais pas pourquoi on y retourne aujourd'hui parce qu'on y est déjà allé hier. Mais ce n'est pas grave parce que j'aime bien aller le voir Monsieur Pépi. Chez lui c'est très grand, au moins deux fois comme ma chambre. Il y a beaucoup de petites machines amusantes, et moi j'ai la chance de m'amuser avec à chaque fois qu'on lui rend visite. Papa, Maman et Monsieur Pépi parlent beaucoup ensemble. Je ne comprends pas tout ce qu'ils disent, ils utilisent beaucoup de mots de grandes personnes. Mais ça m'est égal, parce que pendant qu'ils discutent je suis bien trop occupée à remettre les oiseaux dans les cages et à essayer de réunir les petits lapins et les fleurs ensemble. C'est très difficile parfois, mais avec quelques efforts je finis souvent par $y$ arriver! Et puis c'est beaucoup plus amusant que les exercices que je dois faire à la maison. Mais aujourd'hui c'est différent. Monsieur Pépi ne m'installe pas devant les machines, il ne fait rien de bizarre avec mes lunettes, il me demande juste gentiment de m'assoir entre Papa et Maman et m'explique doucement que demain, je vais me faire opérer.

\subsection{C.T. Charline SORDI}

Il était 7h23 quand mon téléphone sonna, c'était Nicolas.

$$
\begin{aligned}
& \text { « - Troy, j’écoute } \\
& \text { - Il a encore frappé. }
\end{aligned}
$$


- Avenue pasteur, tu ne peux pas louper, la presse est déjà là.

- J'en ai pour dix minutes »

Nos conversations était rarement plus longues que ça, ni plus élaborées pourtant c'était l'un de mes meilleurs collègues et amis. Comme prévu en dix minutes j'étais sur place. Les «vautours» me reconnurent tout de suite et se ruèrent sur moi en hurlant chacun leurs questions. Ils ruinaient ma réputation et salissaient mon nom depuis plus de deux mois, je ne pouvais m'empêcher de penser « quelle bande de connards » en les voyant. Comme d'habitude, c'est Nico qui me sortit de cette galère en passant sous la barrière de sécurité.

- Je t'écoute

- Elsa Francson, 8 ans, fille unique de race blanche, scolarisée en école privée, mère au foyer, famille friquée... bref le topo habituel

- Les lacets ?

- Comme d'hab'

- Merde!

La maison était immense et majestueuse. La décoration et le standing laissaient imaginer le compte en banque des propriétaires. La police scientifique était déjà au travail, à l'affût de la moindre emprunte. Ce qui ne donnerait rien. Je le savais. J'aperçus les parents dans le salon. La mère était assise sur le canapé, regardant dans le vide, elle tenait une tasse de café probablement froid. Le père, debout, essayait de répondre tant bien que mal aux questions de l'inspecteur. Ce qui ne donnerait rien non plus. Je le savais aussi.

Il ne me fut pas bien difficile de trouver la chambre de la petite : le bruit y était le plus intense.

«- Ok merci tout le monde! Maintenant vous sortez !»

Cela ne servait à rien d'être sympathique, surtout dans des moments comme ceux là. La petite n'avait pas été bougée depuis que sa mère avait trouvé le corps ce matin, m'expliqua Nico. Elle était en tenue de cérémonie on aurait dit qu'elle allait à un mariage. Elle avait des jolies boucles blondes qui tombaient sur son visage d'ange et possédait de mignonnes pommettes roses en haut des joues. On aurait dit une vraie poupée déguisée en ange.

Nico me montra ses chaussures : des petites ballerines blanches à lacets.

« Axel ! On n’a pas coffré le bon! Regarde un double nœud comme pour les autres! C'est sa signature, ça ne peut être que lui... c'est la merde! La grosse merde ! »

Sa signature... le tueur qui sévissait dans la région depuis maintenant deux mois en était à sa douzième victime. La presse l'appelait le « tueur d'héritiers », car il s'attaquait seulement aux familles fortunées ne possédant qu'un seul enfant, fille ou garçon. Il les habillait toujours ainsi ou presque. Parfois même, il les maquillait. Le seul élément stable était le double nœud des chaussures dans lequel était coincé une fleur... cette fois c'était une marguerite. Ce détail n'avait pas été révélé à la presse, nous voulions éviter ainsi les « copy-cat ».

«Que fait-on maintenant? Axel! Où vas-tu?»

J'en avais assez vu. Je n'ai pas besoin de regarder cette gamine pendant une heure et me torturer l'esprit. Je sais déjà tout ce qu'il y a à savoir. Deux mois que ce dingue ne laisse aucune trace, aucun indice, j'ai l'impression de courir après un fantôme.

« Je rentre, Camille m'attend.»

Ma fille Camille avait déjà vingt trois ans et depuis deux mois je remerciais dieu qu'elle n'en ait plus dix. Avec tout l'argent que sa mère lui a laissé, le tueur d'héritier aurait pu s'en prendre à elle. 
9h30 : Camille devait être debout, le café frais embaumait le rez-de-chaussée. Je la rejoins donc dans la cuisine. Elle prenait son petit déjeuner. Tout en faisant signe de m'assoir elle me servit une tasse de café et me demandait comment j'allais. Avec elle, nous ne parlions jamais d'enquêtes, jamais du boulot. Après avoir épuisé toutes les banalités de circonstance elle en vint au fait :

« Tu dois venir ce soir. Ce n'est pas une question, ni une proposition papa!

- Je n'aime pas ce mec, Cam, il ne m'aime pas non plus! Alors pourquoi devrait-on se forcer?

- Parce que c'est mon petit ami et que je l'aime. Le jour de notre mariage on fera comment ? tu l'éviteras toute la journée ou ferons-nous une cérémonie sans toi ? comme tu préfères !»

Le mariage... la robe de mariée... Elsa Francson, non ! Il faut que je pense à autre chose. Le sujet de discorde était sans cesse le même. Je détestais cet homme. Dès la première seconde où je l'ai vu je l'ai détesté. Le hasard fait parfois mal les choses car c'est au poste de police que ma fille et lui se sont rencontrés. Camille et lui étaient les témoins du meurtre d'une petite fille. Cela faisait maintenant cinq ans, mais Camille, traumatisée, doit encore y penser tous les jours. Guillaume est un artiste peintre avec, soit disant, beaucoup de talent. Pour moi, il avait seulement celui de me voler ma fille. Mais bien sûr j'y serai à son exposition. Qu'est-ce que je ne ferais pas pour elle...

En tant que bon flic je ne suis jamais en retard. A 19h30 tout juste j'étais à la galerie de mon futur gendre. Evidemment j'étais le premier, Camille n'était pas encore là. Dès mon entrée, Guillaume vint me saluer, non pas par envie mais par pure politesse. J'avais besoin d'un verre, je le lui dis :
« Dans ce genre d'évènement on boit après l'expo non? Pas moyen de boire un verre tout de suite?»

Un sourire apparut sur ses lèvres. Il me désigna l'atelier derrière la porte où il entreposait ses œuvres. La salle était assez grande et très sombre, comment pouvait-il peindre dans ces conditions? Le vin rouge était au fond d'un carton, il était un peu âpre mais il faisait l'affaire. Tout en buvant, je visitais un peu les lieux, les invités n'arriveraient pas avant une bonne vingtaine de minutes et je n'avais pas envie de faire la conversation à Guillaume. Beaucoup de tableaux, et encore des tableaux... jusque là rien d'étonnant. J'en vis même un fait par Camille, elle m'avait dit qu'elle peignait un peu. Manipuler des pinceaux la détendait. Puis mon regard s'arrêta sur un autre tableau, une petite fille en robe de mariée... des ballerines avec un double... mon dieu ! Un, deux, trois... Je comptais les tableaux derrière celui là... il y en avait douze. Mon dieu! Mon dieu! Je jubilais. Je le tiens! Je le tiens! C'est lui j'en suis sûr! Je pris mon téléphone pour prévenir tout de suite Nico. Une lame du parquet grinça.

« Ah! Papa, tu es déjà là, tu les as reconnus? Ce sont mes rêves, mes petits anges...»

Mon regard se figea.

Bon dieu! La signature ? C.T ... Camille Troy... ma fille.

\subsection{CATABASE Benoît Bernay}

Les lignes filent, défilent, s'arrondissent, s'atténuent puis s'envolent.

L'obscurité; le noir toujours le noir, cela fait des heures que j'emprunte seul ces escaliers.

Le vieil homme m'avait prévenu: ceux qui s'enfoncent ne remontent jamais! En même 
temps, je n'ai pas envie de revenir, la descente est suffisamment longue. Je veux atteindre la célèbre terre des rêves: les Champs Élysées! Je crois enfin apercevoir le fond, un glapissement se fait entendre, le voici! Ce magnifique Cerbère, gardant fièrement sa porte. Je suis déçu, il est petit, remue la queue; trois gâteaux suffisent à payer mon passage. Je continue de descendre une pente légère, je vois à mes côtés un flot d'âmes avançant à vive allure, j'en interroge une pour comprendre la source de cette agitation: la barque de Charon accoste, il faut courir pour ne pas la manquer! Suivant la foule, j'arrive en vue du Styx. L'odeur du fleuve me donne la nausée, l'eau semble croupie. Je me dépêche alors de rejoindre le port pour entamer la traversée, il est hors de question de nager. La barque doit être un surnom: devant moi se trouve une grande gondole, à la proue, un squelette enrobé dans une toge. Au vu de la taille de l'embarcation, tout le monde devrait rentrer. La traversée commence Charon pousse sur son immense tige pour nous faire avancer, en quelques coups nous sommes arrivés. Il aurait quand même pu construire un pont, surtout pour si peu. Je descends de la gondole et je reprends la marche.

Un sentier me guide vers une grotte sombre. Dans celle-ci, certaines cavités donnent sur le Tartare, j'aperçois le Pyriphlégéthon avalant des femmes hurlant de douleur. Je verrais bien parmi elles madame Petitbellum, pour toutes les affreuses versions qu'elle m'a imposées durant ma scolarité. Je ne m'inquiète pas, étant simple touriste je ne risque pas d'y aller. Donc le Tartare c'est pour les autres. Sorti des grottes, se dresse, face à moi, un grand amphithéâtre où sont assis sur un podium: Minos, Eaque et .... personne? La troisième place est vide. Étonné, je m'avance et demande où se trouve Rhadamanthe. $\mathrm{Ma}$ question est mal perçue, Minos répond sèchement : en grêve! Eaque tape des mains et crie: Tartare! Je tombe alors dans un trou brûlant.
En bas, je suis accueilli par Alecto hurlante. Heureusement j'ai très vite décelé son handicap : la surdité! Ne m'agaçant pas de ses cris infernaux, je lui explique en gesticulant que je suis juste un vacancier des enfers qui a énervé les juges Minos et Eaque. Elle me crie que pour remonter, il faut persuader un titan de s'occuper de moi. Les titans; de mémoire ceux qui croupissent au Tartare n'aiment pas trop les humains. Je vais plutôt essayer de voir si les hécatonchires peuvent BEM161m'aider. En effet, j'aperçois Briarée à proximité, je m'empresse de lui expliquer l'erreur juridique dont je suis victime. Celui-ci rigole et m'explique que les juges se sont récemment disputés sur un point existentiel: estce-qu' écraser la cocotte en chocolat de son petit frère, le jour de pâques, est passible d'exil au Tartare ou non? Si je veux atteindre les Champs Élysées, je dois trouver Rhadamanthe et lui donner LA réponse à cette question.

Confiant dans la réussite de mon épopée, Briarée me dépose dans l'Érèbe et je pars à la recherche de Rhadamanthe. Je m'en vais loin du sentier des âmes, interrogeant les différents démons. Une succube m'aiguille, le juge serait remonté dans le monde des vivants pour constater l'ampleur des dégâts provoqués par une cocotte en chocolat écrasée. Apparemment on pourrait le contacter, en utilisant l'œil des Grées. Je remercie la charmante diablesse et poursuit les sœurs Grées. Celles-ci se trouvant temporairement aux enfers, la tâche n'en est que plus facile. Une fois arrivé chez elles, je m'annonce. Elles boivent le thé, elles m'invitent. Je me méfie: je connais leur réputation, car de toutes les épopées, celle de Persée est une de mes préférées. Une fois de plus je m'aperçois que les écrivains étoffent leurs héros, les trois Grées me prêtent volontiers leur œil. Je contacte le juge. Heureux qu'un mortel accepte de donner son point de vue, il me convie à en débattre avec Minos et Eaque. 
J'arrive dans un tribunal désert. Aucune âme dans les gradins, seuls les trois juges sont là. Je me présente devant eux. Immédiatement Rhadamanthe m'explique l'origine exacte de la dispute : Eaque pense qu'il faut envoyer l'écraseur de cocotte au Tartare, Minos non, et lui reste sans opinion. II en revient à moi, simple mortel, de donner mon avis. J'explique alors que briser une cocotte en chocolat ne constitue pas nécessairement un acte mauvais. Tout dépend de l'intention, si on veut distraire l'assemblée par cette surprenante destruction, c'est blâmable mais pas condamnable, de plus, on pourra toujours consoler le petit frère en lui offrant une nouvelle cocotte en chocolat. Mais si on souhaite juste torturer le petit frère alors oui, le Tartare doit être la punition. Rhadamanthe paraît apprécier mon raisonnement; approuvant mes dires, il me laisse partir en direction des Champs Élysées, peu de chemin m'en sépare encore. Je sens d'ici l'odeur d'un excellent repas qui se prépare, j'aperçois les champs en fleurs aux couleurs printanières, je ferme alors les yeux pour mieux ressentir ce nouveau monde.

Un choc me ramène en sursaut. Les lignes sont troubles: j'avais posé mes lunettes. Devant moi se tient l'origine de ce rappel soudain: Madame Petitbellum, une règle à la main. Malgré tout le chemin parcouru, je n'ai pas encore fini ma catabase.

\section{Projet lecture Génie Electrique 2 année}

\section{3 .1 Listes de romans}

\section{9/2010}

Thème : «Qui suis-je ? »

1. Jeanne, Jeanne, Jeanne / Emmanuel Adely. Stock, 2000. - 331p.

2. Talk, Talk / T.C. Boyle. - Grasset, 2007.$439 p$.

3. Dans le miroir Suivi de Appassionata / André Brink. - Actes Sud, 2009. - 214p.
4. Histoire de la femme cannibale / Maryse Condé. - Gallimard (Editions), 2005. - 351p.

5. Le Dernier de son espèce / Andréas Eschbach. - L'Atalante, 2006. - 292p.

6. Middlesex / Jeffery Eugenides. - L'Olivier, 2003. - 678p.

7. Au Pays des vivants / Nicci French. - Pocket, 2006. - 462p.

8. Monsieur Ripley / Patricia Highsmith. - LGF, 2000. - 318p.

9. Double miroir / Jonathan Kellerman. - Pocket, 2004. - 450p.

10. L'Homme qui voulait vivre sa vie / Douglas Kennedy. - Pocket, 1999. - 498p.

11. La Mémoire dans la peau / Robert Ludlum. LGF, 2007. - 666p.

12. Usurpation d'identité / David Morell. - LGF, 1996. -

13. Le pont de l'âme, Thierry Pfister/ Albin Michel avril 2009

14. L'intérieur de la nuit, Léonora Miano / Pocket, 2006.

15. Et Le coucou, dans l'arbre, se rit de l'époux / Percy Kemp. - Albin Michel, 2005. - 280p.

16. Eldorado / Gaudé, Laurent. - Actes-Sud, 2006. $-237 \mathrm{p}$.

17. Des Fleurs pour Algernon/ Daniel Keyes. J'ai lu, 2001. - 252p.

18. Dr Jekyll and Mr Hyde / R. L. Stevenson. J'ai lu, 2003. - 92p.

19. Indian Killer / Alexie SHERMAN. - 10/18, 2000. - 415p.

20. Kafka sur le rivage / Murakami, Haruki. Belfond, 2006. $-618 \mathrm{p}$.

\section{6/2007}

"Sciences, techniques, technologies et fiction "

1 Dracula / Bram Stoker. - J'ai lu, 2005. - 574p.

2 Les Piliers de la terre / Ken Follett. - LGF, 1992. 1074 p.

3 La Pyramide / Ismaïl Kadaré. - LGF, 1994. - 158p.

4 La Muraille de Chine / Takeshi Kaiko. - Picquier, 2004. - 108p.

5 Le meilleur des mondes / A. Huxley . - Pocket, 2005. - 284p.

6 Oncle Pétros et la conjecture de Goldbach / Apostolos Doxiadis. - Le Seuil, 2002. - 205p.

7 Code Tetraktys / Armand Herscovici. - Le Seuil, 2002. - 348p.

8 Zero / Denis Guedj. - Robert Laffont, 2005. - 312p.

9 Des fleurs pour Algernon/ Daniel Keyes. - J'ai lu, 2001. - 252p. - 
10 SOS Sosie, André Chauchat. - Le Pommier, 2002. 190p. -

11 Le rendez-vous de Vénus, Jean-Pierre Luminet. LGF, 2001. - 345p.

12 De la Terre à la lune / Jules Verne. - Elcy, 2005. 190p.

13 Rendez-vous avec Rama / A.C. Clarke. - J'ai lu, 2002. -253 p. -

14 Pourquoi j'ai mangé mon père ?/ Roy Lewis. Babel, 2002. - 171p.

15 La Nuit des temps / Barjavel . -Pocket, 2004. $397 \mathrm{p}$.

16 Lentement s'empoisonnent, Joëlle Wintrebert. Flammarion, 1999. - 233p.

17 Mordoc / Patricia Cornwell. - LGF, 1999. - 346p.

18 Avicenne ou la route d'Ispahan, Gilbert Sinoue. Gallimard, 1990. - 532p.

19 I, Robot / Isaac Asimov. - J'ai lu, 2004. - 320p.

20 Blade runner / Dick. - J'ai lu, 2003. - 250p.

Le Dernier de son espèce / Andréas Eschbach. -

L'Atalante, 2006. - 292p. -

22 Frankenstein, Mary Shelley. - J'ai lu, 2005. - 315p. 23 Dr-Jekyll-and-Mr-Hyde / R. L. Stevenson. - J'ai lu, 2003. -92 p. -

24 Ferdinand et les Iconoclastes, Valérie Tong Cuong. - Grasset et Fasquelle, 2003. - 369p.

25 Cyberdanse macabre, Richard Canal. - Flammarion, 1999. - 241p. -

26 Neuromancien / William Gibson. - J'ai lu, 2001. $319 \mathrm{p}$.

27 Un paysage du temps / Gregory Benford. -

Gallimard, 2001. - 629p. -

28 La Machine à explorer le temps / H. G. Wells. Gallimard, 2002. - 166p. -

29 Solaris / Stanislas Lem. - Gallimard, 2003. -320p. -

30 Les Fourmis / Bernard Werber. - J'ai lu, 1993. $343 p$.

31 Le Jour des Triffides / John Wyndham. - Terre de brume, 2005. 256p. 

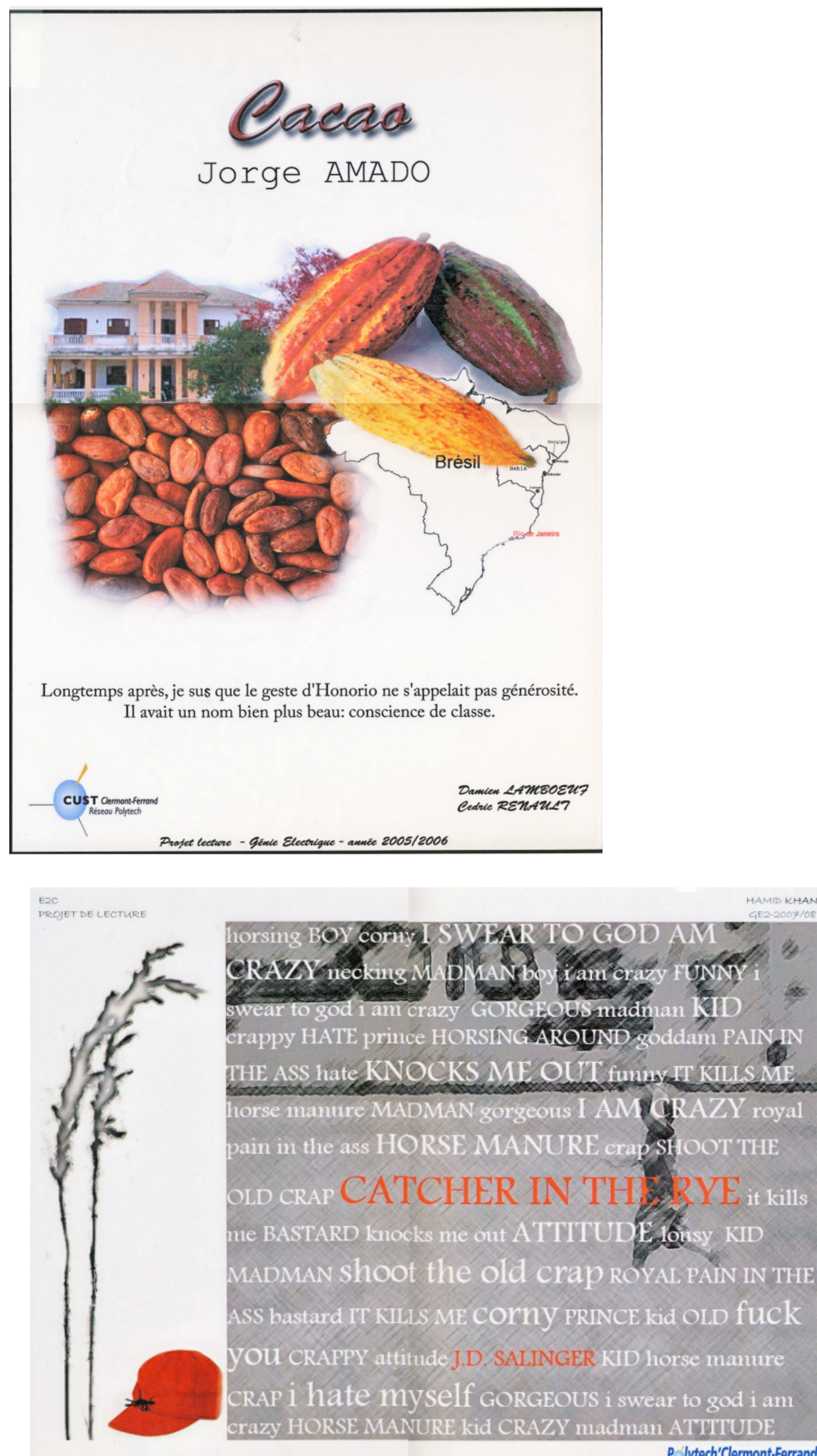


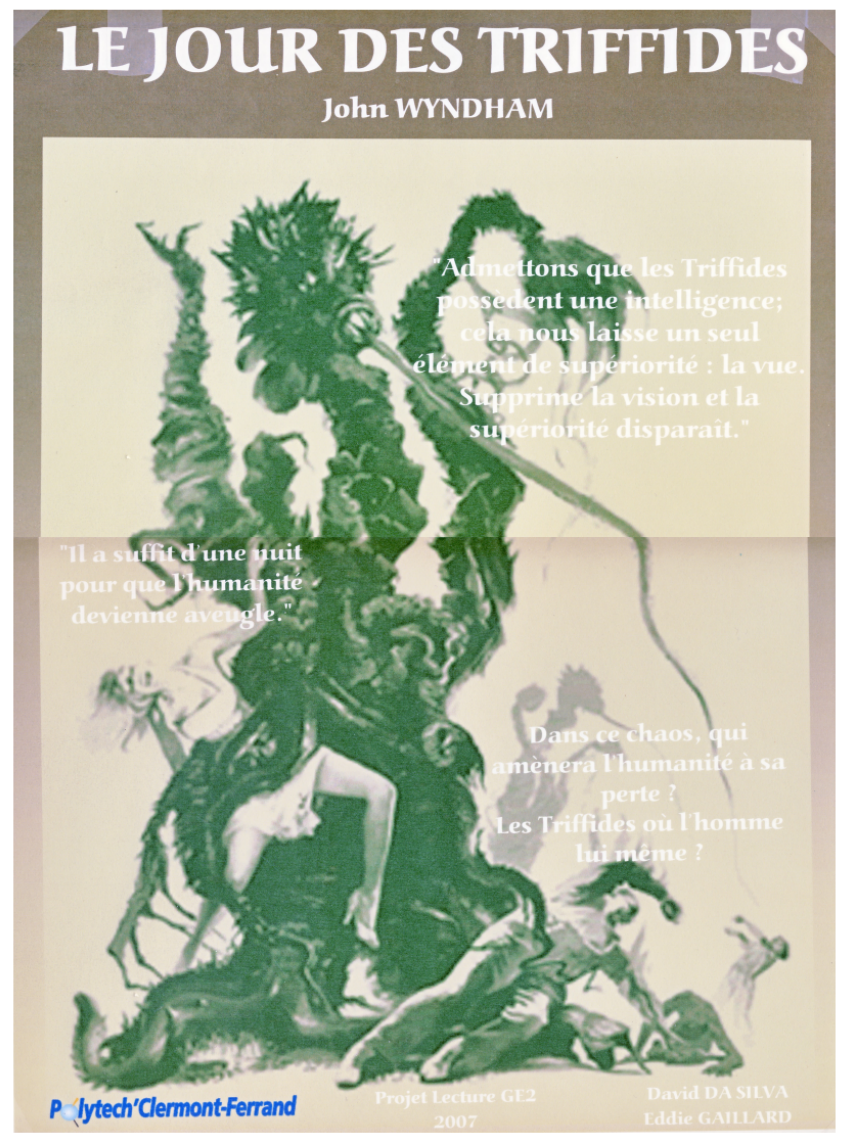

\section{Gil Courtemanche}

\section{Un dimanche à la piscine à rrigali}

C'est parfois au milieu de la barbarie et des manipulations politiques que naissent les plus belles histoires.

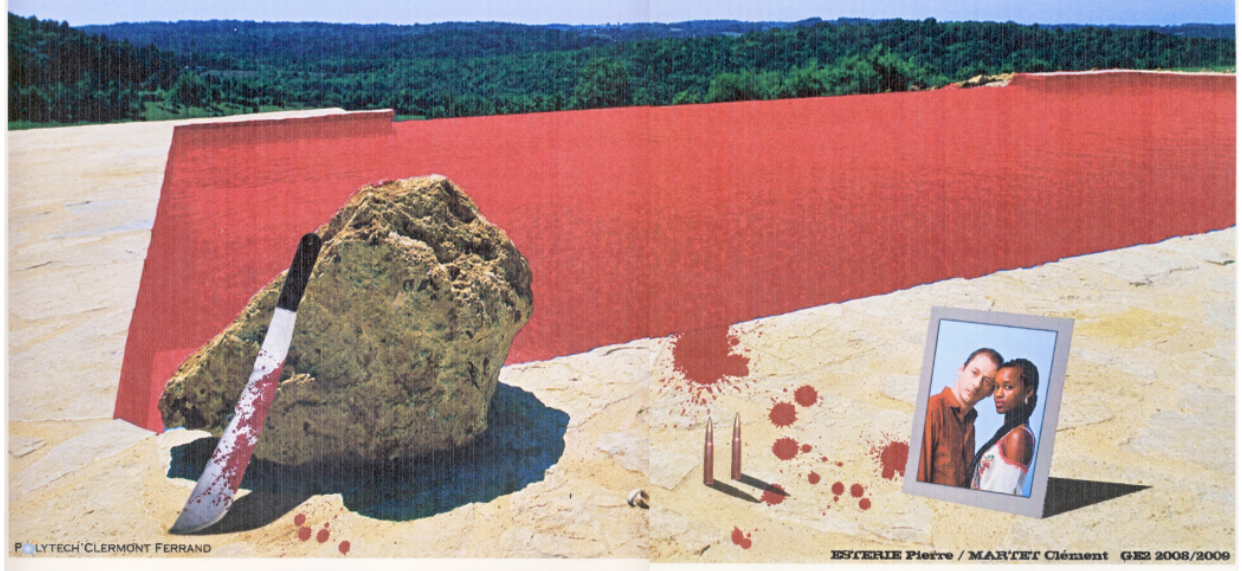

